\title{
Poly(amidoamine) Dendrimers Increase Antifungal Activity of Clotrimazole
}

\author{
Katarzyna Winnicka, ${ }^{*, a}$ Katarzyna Sosnowska, ${ }^{a}$ Piotr Wieczorek, ${ }^{b}$ Pawel Tomasz SAcha, ${ }^{b}$ and \\ Elzbieta TRYNISZEWSKA ${ }^{b}$ \\ ${ }^{a}$ Department of Pharmaceutical Technology, Faculty of Pharmacy, Medical University of Bialystok; and ${ }^{b}$ Department of \\ Microbiological Diagnostics, Faculty of Pharmacy, Medical University of Bialystok; Kilinskiego 1, 15-089 Bialystok, \\ Poland. Received February 9, 2011; accepted March 30, 2011
}

\begin{abstract}
Clotrimazole (CLO) is a local imidazolic antifungal agent. A major problem associated with the successful formulation of effective dosage forms containing CLO is its poor aqueous solubility, which presents a hindrance for the local availability of CLO and limits the effective antifungal therapy. In the present study, the effects of various concentrations of poly(amidoamine) (PAMAM) dendrimers generation 2 (G2) and generation 3 (G3) with amine (PAMAM-NH $)$ or hydroxyl surface groups (PAMAM-OH) on aqueous solubility and antifungal activity of CLO were studied. The obtained results showed that all tested PAMAM dendrimers improved the solubility of CLO and the more potent were PAMAM- $\mathrm{NH}_{2}$ dendrimers. The increase in solubility of CLO was highest at dendrimer concentration of $10 \mathrm{mg} / \mathrm{ml}$. Microbiology studies indicated that only PAMAM- $\mathrm{NH}_{2}$ dendrimers significantly increased the antifungal activity of CLO (a 4-32-fold increase in the antifungal activity compared to pure CLO) and the most potent was dendrimer PAMAM- $\mathrm{NH}_{2}$ G2. These observations indicate that PAMAM dendrimers might be considered as potential carriers of $\mathrm{CLO}$ and provide further impetus to evaluate these polymers for use in basic drug delivery studies and to design semisolid dosage forms based on dendrimers with antimicrobial drugs, like CLO.
\end{abstract}

Key words poly(amidoamine) dendrimer; clotrimazole; aqueous solubility; antifungal activity

Clotrimazole (1-2-chlorophenyl-diphenylmethyl)-1-4-imidazole (CLO) is an azole-type antifungal agent (Fig. 1) that is known to have topical activity against pathogenic dermatophytes and yeasts. ${ }^{1,2}$ It is primarily used locally in the treatment of vaginal and skin infections and shares with econazole and miconazole the first choice status for topical treatment of candidiasis. ${ }^{3,4)}$ CLO inhibits biosynthesis of ergosterol, the key sterol in most pathogenic fungi and increases cellular permeability. Permeability changes result in loss of essential precursors, metabolites, and ions, thus inhibiting macromolecular synthesis. ${ }^{2)}$ However, the clinical use of CLO has some practical disadvantages mainly due to its poor water solubility. Therefore, the development of novel carriers for CLO could lead to improve its solubility and permeability. It has been attempted to improve the solubility of CLO by microcapsules, ${ }^{5)}$ liposomes ${ }^{6}$ and cyclodextrins complexation. $^{\text {) }}$

Recently a new class of macromolecules, the dendrimers, has received much attention for their ability to solubilise water insoluble drugs and their ability to promote the transport of drugs across biomembranes. ${ }^{8-10)}$ Dendrimers are core-shell nanostructures with precise architecture and low polydispersity, which are synthesized in a layer-by-layer fashion (expressed in "generations") around a core unit, resulting in high level of control over size, branching points and surface functionality. They consist of a central core and several generations of three-dimensional branches which result in a large number of functionalized end groups at the

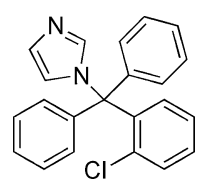

Fig. 1. Chemical Structure of Clotrimazole Molecule surface. ${ }^{11)}$ Poly(amidoamine) (PAMAM) dendrimers (Fig. 2) are one of the most studied dendritic polymer class today. They possess a number of favourable properties including well-defined chemical structure, globular shape, low polydispersity index (close to 1.0), biocompatibility, and perfect solubility in a large number of solvents, particularly in water. ${ }^{12)}$ Moreover, their spherical architecture provides a compact structure $(1-10 \mathrm{~nm})$ for transport across biological membranes. The high level of control over the dendritic architecture (size, branching density, surface functionality) makes PAMAM dendrimers ideal carriers for enhanced solubility of poorly water-soluble drugs. ${ }^{13)}$ Moreover, PAMAM dendrimers are being considered as additives in several routes of administration, including intravenous, oral, transdermal, and ocular. $^{12,14)}$ Some of dendrimers themselves show pharmaceutical activity (anticancer, anti-inflammatory, antimicrobial), providing the opportunity for combination therapy in which the dendrimers serve as the drug carrier and simultaneously as an active part of the therapy. 12,15$)$

Since, to our knowledge, there are no studies devoted to the solubilization of CLO in the presence of dendrimers, the present study was aimed to evaluate the influence of PAMAM dendrimers on its solubility and antifungal activity. The goal of this work was to study the effect of concentration, generation and type of surface functional groups on the solubility of CLO and to investigate antifungal activity of CLO in the presence of PAMAM dendrimers with amine and hydroxyl surface groups.

\section{MATERIALS AND METHODS}

Materials Clotrimazole was a gift from Aflofarm (Pabianice, Poland). PAMAM dendrimers generation 2 (G2) and generation 3 (G3) with amine and hydroxyl surface groups, RPMI 1640 medium, dimethyl sulfoxide (DMSO) were pro- 
A)

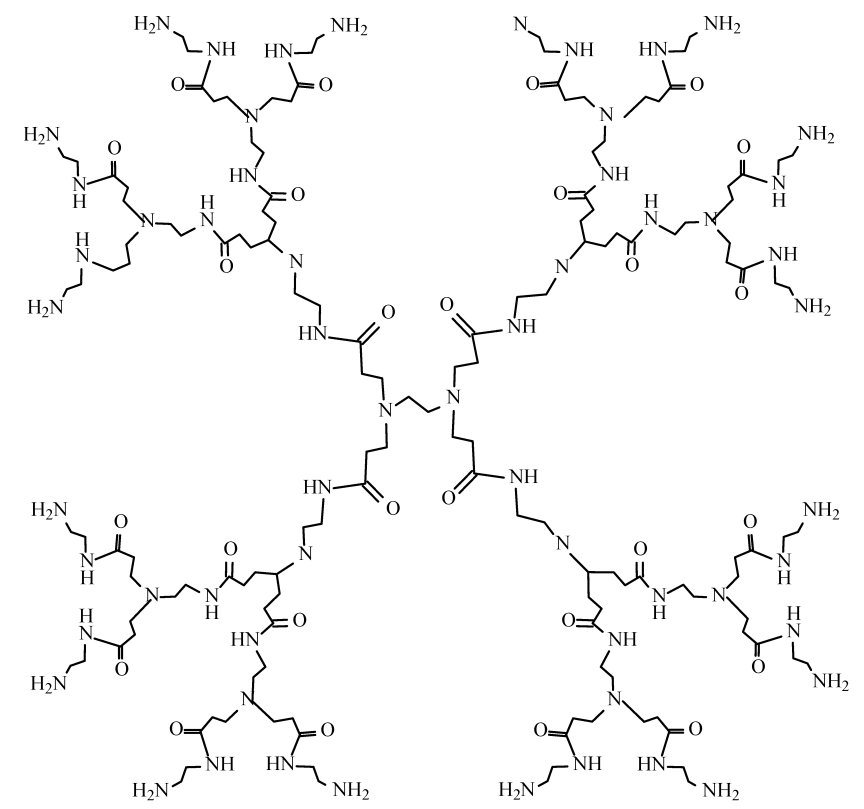

B)

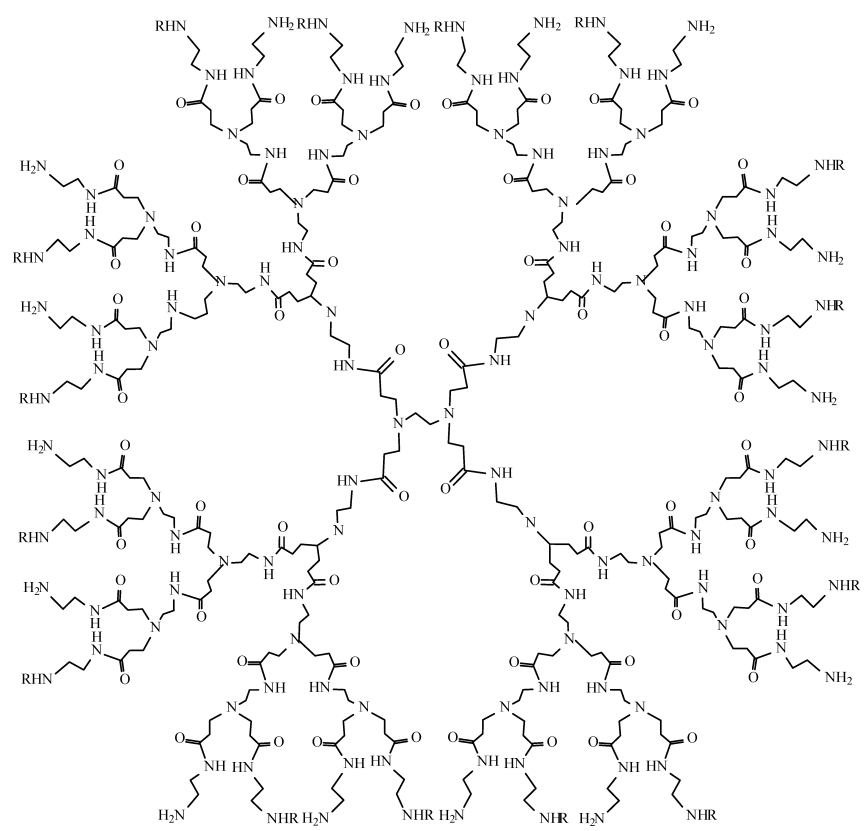

Fig. 2. Chemical Structure of PAMAM-NH $\mathrm{H}_{2}$ Dendrimer Generation 2 (A) and Generation 3 (B)

vided by Sigma Aldrich (St. Louis, U.S.A.), as were most other chemicals and buffers used. Stock cultures of Candida parapsilosis ATCC 22019 were purchased from Microbiologics (St. Cloud, U.S.A.). Methanol HPLC grade was obtained from Merck (Darmstadt, Germany). Mixed cellulose esters $(\mathrm{HA})$ membrane filters $(0.45 \mu \mathrm{m})$ were received from Millipore (Billerica, U.S.A.).

Solubility Studies The solubility of CLO was determined by using shake-flask method. Briefly, an excess amount of CLO was added to each vial containing $5 \mathrm{ml}$ of the selected vehicle (water or $5 \mathrm{mg} / \mathrm{ml}, 10 \mathrm{mg} / \mathrm{ml}$, and $20 \mathrm{mg} / \mathrm{ml}$ solutions of G2 and G3 PAMAM dendrimers with amine or hydroxyl surface groups). Mixtures were mechani- cally shaken for $48 \mathrm{~h}$ at $25 \pm 0.5^{\circ} \mathrm{C}$ and allowed to stand for $24 \mathrm{~h}$ to attain equilibrium. Then mixtures were centrifuged at $3000 \mathrm{rpm}$ for $15 \mathrm{~min}$, followed by filtration through HA membrane filter $(0.45 \mu \mathrm{m})$, diluted appropriately with the methanol and analyzed by HPLC method at $210 \mathrm{~nm} .{ }^{16,17)}$

HPLC Analysis The solubility of CLO was determined by the HPLC system Agilent Technologies 1200 equipped with a G1312A binary pump, a G1316A thermostat, a G1379B degasser and a G1315B diode array detector (Agilent, Waldbronn, Germany). Data collection and analysis were performed using Chemstation 6.0 software. Separation was achieved on a Zorbax Eclipse XDB-C18, $4.6 \times 150 \mathrm{~mm}$, $5 \mu \mathrm{m}$ column (Agilent, Waldbronn, Germany). Mobile phase was methanol-phosphate buffer $\mathrm{pH} 7.4(4: 1, \mathrm{v} / \mathrm{v})$, the flow rate was $1.0 \mathrm{ml} / \mathrm{min}$ and UV detection was performed at a wavelength of $210 \mathrm{~nm} .{ }^{16,17)}$ The column temperature was maintained at $25^{\circ} \mathrm{C}$. For injection into the HPLC system $20 \mu \mathrm{l}$ of sample was used. All reagents used for analysis were HPLC grade.

Test Organisms The antifungal activity was evaluated against yeast cultures Candida parapsilosis ATCC 22019, and against susceptible and drug resistant clinical strains belonging to the species C. albicans, C. glabrata, C. krusei, $C$. dubliniensis and C. tropicalis. Candida strains were isolated from selected patients with candidiasis, identified morphologically, ${ }^{18)}$ and stored in potato dextrose broth at $-70^{\circ} \mathrm{C}$. Prior to antifungal susceptibility testing, each isolate was inoculated on potato dextrose agar plates to ensure optimal growth characteristics and purity. Then yeast cells were suspended in saline and adjusted spectrophotometrically to RPMI 1640 medium.

Antifungal Agent CLO $>99 \%$ pure was obtained from Aflofarm (Pabianice, Poland) as a fluffy white powder. CLO is insoluble in water but dissolves in organic solvents. ${ }^{19)} \mathrm{A}$ stock solution of $5120 \mu \mathrm{g} / \mathrm{ml}$ was prepared by dissolving CLO in sterile DMSO and in $10 \mathrm{mg} / \mathrm{ml}$ PAMAM dendrimers G2 and G3 with amine or hydroxyl surface groups. These solutions were stored in dark glass bottles and were used for all experiments in this study. Series of double diluting solutions of above compounds were prepared in RPMI 1640 medium (with L-glutamine, without sodium bicarbonate and buffered to $\mathrm{pH} 7.0$ with 3 -(n-morpholino)propanesulfonic acid) obtaining the final concentration of CLO in the range from 512 to $0.004 \mu \mathrm{g} / \mathrm{ml}$. Solutions of PAMAM dendrimers and DMSO were also evaluated in the absence of CLO.

Broth Microdilution Method Antifungal activity was tested by using broth microdilution method according to the Clinical and Laboratory Standards Institute (CLSI) guidelines. ${ }^{20,21)}$ The medium used for susceptibility testing was RPMI 1640 with L-glutamine, without sodium bicarbonate and buffered to $\mathrm{pH} 7.0$ with 3-(n-morpholino)propanesulfonic acid. The initial density of Candida was approximately $2-5 \times 10^{6}$ colony forming units $(\mathrm{CFU}) / \mathrm{ml}$. Inoculums of fungi (density of 0.5 in McFarland scale) were prepared in sterile solution of $0.9 \% \mathrm{NaCl}$. Then tested strains were suspended in RPMI 1640 medium to give a final density of $5 \times 10^{4} \mathrm{CFU} / \mathrm{ml}$. Solutions of CLO, CLO in PAMAM dendrimers and suspensions of fungi were inoculated onto microtiter plates. The growth control, sterility control and control of antifungal compounds were used. Plates were incubated under normal atmospheric conditions at $35^{\circ} \mathrm{C}$ for $48 \mathrm{~h}$, 
and next minimum inhibitory concentration (MIC) values have been determined. The MIC was defined as the lowest concentration required to arrest the growth of the fungi. For determination of minimum fungicidal concentration (MFC), a $0.01 \mathrm{ml}$ aliquot of the medium drawn from the culture tubes showing no macroscopic growth at the end of the $24 \mathrm{~h}$ culture was subcultured on potato dextrose agar plates to determine the number of vital organisms and incubated further for appearance of yeast-like growth. The MFC was defined as the lowest concentration of the agent at which no colonies are observed. ${ }^{22}$ All MIC and MFC experiments were repeated three times. The stock solution of pure CLO was used as positive control. Solvent and media controls were used for reference.

Data Analysis The results were analysed by analysis of variance (ANOVA) and multiple comparison were done to check statistical significance. The statistical significance between means was verified by Scheffe's comparison test accepting $p<0.05$ as significant.

\section{RESULTS AND DISSCUSION}

A major problem associated with the successful formulation of effective dosage forms containing CLO is its poor aqueous solubility $0.49 \mu \mathrm{g} / \mathrm{ml}^{23)}$ Poor water solubility of CLO presents a hindrance for the local availability of CLO and limits the effective antifungal therapy.

It has been a constant ambition to formulate and optimize an efficient delivery systems for hydrophobic drugs. Polymers have been widely investigated as carriers of both covalently bound and physically entrapped drug molecules to improve their water solubility, decrease their toxicity, increase permeability and increase the site-specific delivery of drugs. ${ }^{24)}$ PAMAM dendrimers with uniform and well-defined particle size and shape are of eminent interest in biomedical applications because of their ability to cross cell membranes. Non-polar cavities in PAMAM dendrimers in combination with their hydrophilic exterior surface make them capable of encapsulating hydrophobic drug molecules. ${ }^{8,9,13)}$ These noncovalent inclusions offer a variety of physicochemical advantages over the free drug molecules including the possibility of enhanced water solubility and drug stability. The quest molecules can be encapsulated in the hydrophobic cavities or can be attached to the functional groups on the surface of the dendrimer. Dendrimers enhance the solubility of hydrophobes due to hydrophobic interactions, hydrogen bonding and electrostatic interaction between terminal functional groups of the dendrimers and hydrophobes. ${ }^{25-27)}$

The results of the solubility studies are shown in Fig. 3. It is evident that all PAMAM dendrimers enhance solubility of CLO. The apparent solubility of CLO increased linearly as a function of PAMAM concentration over the range 0 $10 \mathrm{mg} / \mathrm{ml}$. The increase in solubility was highest at dendrimer concentration of $10 \mathrm{mg} / \mathrm{ml}$. At higher, $20 \mathrm{mg} / \mathrm{ml} \mathrm{con-}$ centrations, the solubility increase was definitely lower. In the case of PAMAM-NH $\mathrm{N}_{2}$ G3 dendrimer, the solubility of CLO was found to be enhanced approximately 6.7-fold (from 0.38 to $2.55 \mu \mathrm{g} / \mathrm{ml}$ ). Among the PAMAM dendrimers used in the study, PAMAM-NH $\mathrm{H}_{2} \mathrm{G} 3$ exhibited the highest solubilising potential for CLO followed by PAMAM-NH $\mathrm{H}_{2}$ 2, PAMAM-OH G3, and PAMAM-OH G2. As PAMAM den-
A)

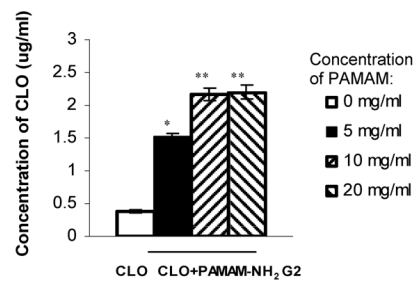

C)

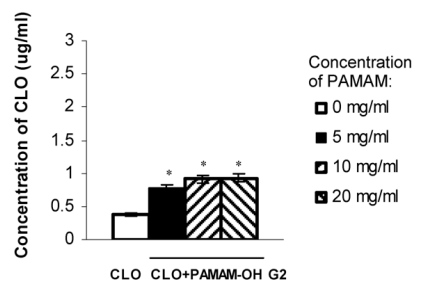

B)

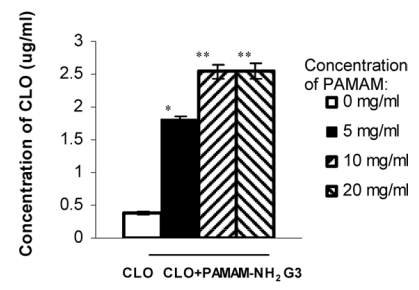

D)

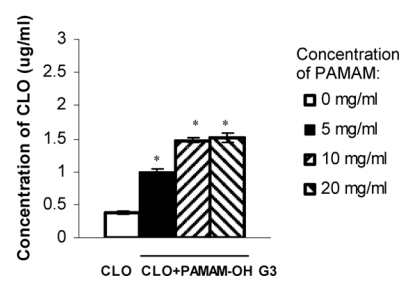

Fig. 3. Solubility of Clotrimazole in the Presence of Various Concentrations of PAMAM- $\mathrm{NH}_{2}$ Generation 2 (A), PAMAM- $\mathrm{NH}_{2}$ Generation 3 (B), PAMAM-OH Generation 2 (2) and PAMAM-OH Generation 3 (D)

Mean values from three independent experiments done in duplicate are presented. $* p<0.05$ and $* * p<0.01$

drimers possess empty internal cavities, small organic molecule drugs like CLO may be encapsulated into the dendrimer's interior void space, while larger molecules preferably adsorb onto the dendrimer surface. The higher generation and more surface groups, the more molecules of drug can interfere by electric interactions. CLO as a weak base $\left(\mathrm{p} K_{\mathrm{a}}=6.12\right)^{28)}$ is rather encapsulated than attached to dendrimers' amine or hydroxyl end groups. Driving forces for CLO-PAMAM interactions are hydrophobic interactions between dendrimer cavities and drugs, and hydrogen bonds between tertiary amines within the PAMAM core and drug molecules.

The results concerning the in vitro antifungal activity of CLO in $10 \mathrm{mg} / \mathrm{ml}$ solutions of PAMAM-NH $\mathrm{N}_{2}$ and PAMAM$\mathrm{OH}$ dendrimers obtained by broth dilution method are listed in Tables 1 and 2. The MIC and MFC values significantly indicate that PAMAM- $\mathrm{NH}_{2}$ dendrimers increased the antifungal activity of CLO against all the Candida cultures in the most potent way. CLO in solution of PAMAM-NH $\mathrm{H}_{2}$ was 4-32-fold more potent than pure CLO (e.g. in culture C. albicans $1005279 / 1 / 10 \mathrm{MIC}$ value was $4 \mu \mathrm{g} / \mathrm{ml}$ and $32 \mu \mathrm{g} / \mathrm{ml}$, and MFC was $4 \mu \mathrm{g} / \mathrm{ml}$ and $128 \mu \mathrm{g} / \mathrm{ml}$ for CLO in solution of PAMAM-NH $\mathrm{N}_{2}$ G2 and pure CLO, respectively). This enhanced antifungal activity could not be contributed to the dendrimer itself as PAMAM G2 and G3 are known to displayed cytotoxic activity at a much higher concentration. ${ }^{29)}$ The size of the dendrimer affects its ability to penetrate the cell membrane and therefore its antimicrobial activity. In this study, PAMAM- $\mathrm{NH}_{2}$ G2 was found to be significantly more active than PAMAM-NH $\mathrm{N}_{2}$ G3. Similarly, PAMAM G3 dendrimers were more effective antibacterial agents than PAMAM G5, and lower generation of carbosilane dendrimers was found to have more antimicrobial activity than the higher one. ${ }^{30)}$

It was found that the most potent was CLO in PAMAM$\mathrm{NH}_{2}$ dendrimers. It may be caused by the highest rate of the cell uptake for the cationic dendrimer, followed by anionic 
Table 1. Minimum Inhibitory Concentration (MIC) (in $\mu \mathrm{g} / \mathrm{ml}$ ) of Clotrimazole in the Presence of PAMAM-OH and PAMAM-NH ${ }_{2}$ Dendrimers Generation 2 (G2) and Generation 3 (G3)

\begin{tabular}{|c|c|c|c|c|c|}
\hline \multirow{3}{*}{ Fungal species } & \multicolumn{5}{|c|}{$\operatorname{MIC}(\mu \mathrm{g} / \mathrm{ml})$} \\
\hline & \multirow{2}{*}{ CLO } & \multicolumn{2}{|c|}{$\mathrm{CLO}+\mathrm{PAMAM}-\mathrm{OH}$} & \multicolumn{2}{|c|}{$\mathrm{CLO}+\mathrm{PAMAM}^{-\mathrm{NH}_{2}}$} \\
\hline & & G2 & G3 & G2 & G3 \\
\hline Candida albicans 1005279/1/10 & 32 & 32 & 32 & 4 & 16 \\
\hline Candida albicans 1005275/1/10 & 64 & 32 & 32 & 16 & 16 \\
\hline Candida albicans 1103055/11 & 1 & 0.5 & 0.5 & 0.25 & 0.5 \\
\hline Candida dubliniensis 1103124/11 & 0.125 & 0.125 & 0.125 & 0.125 & 0.125 \\
\hline Candida glabrata 1005275/2/10 & 64 & 64 & 64 & 16 & 32 \\
\hline Candida glabrata 1102890/11 & 0.5 & 0.5 & 0.5 & 0.25 & 0.5 \\
\hline Candida krusei 1103055/11 & 0.016 & 0.016 & 0.016 & 0.008 & 0.008 \\
\hline Candida tropicalis $1005328 / 10$ & 32 & 32 & 32 & 4 & 8 \\
\hline Candida parapsilosis ATCC 22019 & 0.64 & 0.64 & 0.64 & 0.32 & 0.32 \\
\hline
\end{tabular}

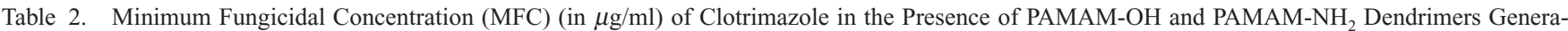
tion 2 (G2) and Generation 3 (G3)

\begin{tabular}{|c|c|c|c|c|c|}
\hline \multirow{3}{*}{ Fungal species } & \multicolumn{5}{|c|}{$\operatorname{MFC}(\mu \mathrm{g} / \mathrm{ml})$} \\
\hline & \multirow{2}{*}{ CLO } & \multicolumn{2}{|c|}{$\mathrm{CLO}+\mathrm{PAMAM}-\mathrm{OH}$} & \multicolumn{2}{|c|}{$\mathrm{CLO}+\mathrm{PAMAM}^{-\mathrm{NH}_{2}}$} \\
\hline & & G2 & G3 & G2 & G3 \\
\hline Candida albicans 1005279/1/10 & 128 & 128 & 128 & 4 & 8 \\
\hline Candida albicans 1005275/1/10 & 128 & 128 & 64 & 4 & 16 \\
\hline Candida albicans 1103055/11 & 32 & 32 & 32 & 2 & 4 \\
\hline Candida dubliniensis 1103124/11 & 32 & 32 & 32 & 8 & 32 \\
\hline Candida glabrata 1005275/2/10 & 256 & 256 & 128 & 16 & 32 \\
\hline Candida glabrata 1102890/11 & 32 & 32 & 32 & 2 & 4 \\
\hline Candida krusei 1103055/11 & 0.5 & 0.25 & 0.25 & 0.125 & 0.25 \\
\hline Candida tropicalis 1005328/10 & 128 & 128 & 128 & 16 & 64 \\
\hline Candida parapsilosis ATCC 22019 & 1.0 & 1.0 & 1.0 & 0.5 & 0.5 \\
\hline
\end{tabular}

and neutral dendrimers. ${ }^{31)}$ The surface charge of the dendrimers influences their cell uptake. The cationic dendrimers may be taken up inside the cells by adsorptive endocytosis through interaction with the negatively charged proteoglycans in the cell membrane. ${ }^{31)}$ The enhanced in vitro antifungal activity of CLO in PAMAM- $\mathrm{NH}_{2}$ dendrimers solutions may be attributed to enhanced penetration of CLO through fungal cell walls to inhibit ergosterol synthesis. PAMAM dendrimers with amine surface functional groups, particularly primary amine functional group could penetrate through the cell membrane of microorganisms, mainly due to their strong hydrogen bond donor characteristic properties toward biomolecules. ${ }^{32)}$ Dendrimers are able to destabilize the membrane by creating small holes in a bilayer. Such holes facilitate dendrimer transport across cell membranes and are dependent on dendrimer structure and surface charge, with cationic dendrimers being the most active. ${ }^{33)}$ DSC and Raman spectroscopy investigations showed that dendrimers caused a concentration dependent increase in the lipid membrane fluidity as they strongly interact with both the lipophilic tails and polar head groups of phospholipids. ${ }^{34)}$ Leroueil et al. employed atomic force microscopy to determine the common mechanism of interaction of cationic nanoparticles (PAMAM dendrimers, polyethyleneimine and diethylaminoethyl-dextran, inorganic particles $\mathrm{Au}-\mathrm{NH}_{2}$, $\mathrm{SiO}_{2}-\mathrm{NH}_{2}$ ) with lipids and physical disruption of lipid membranes. ${ }^{35)}$ They found that these polycationic nanoparticles interacted with biological membranes and induced cell disruption by formation of nanoholes, membrane thinning, and/or membrane erosion. ${ }^{35)}$ Initial interaction between cationic macromolecules and the negatively charged cell membrane is mediated by electrostatic interactions. Interactions with membrane proteins and phospholipids seem to disturb membrane structure and function. Arnold et al. suggested that poly(L-lysine) triggers cellular efflux of organic and inorganic substances proportional to its membrane adsorption. ${ }^{36)}$ Amine-terminated G7 PAMAM dendrimers $(10-100 \mathrm{nM})$ were observed to form holes of $15-40 \mathrm{~nm}$ in diameter in aqueous, supported 1,2-dimyristoyl-sn-glycero-3phosphocholine (DMPC) lipid bilayers. ${ }^{32)}$ G5 amine-terminated dendrimers did not initiate hole formation but expanded holes at existing defects. Interestingly, acetamide-terminated G5 PAMAM dendrimers did not cause hole formation in this concentration range. ${ }^{32)}$ Amine-terminated dendrimers are protonated in neutral water and therefore can be anticipated to have a substantial electrostatic interaction with the DMPC lipid. However, it is clear that there is a large difference between living cells and model membranes. This is mainly because dendrimers need to cross the cell wall, an external barrier, before reaching the cytoplasmic membrane. Many pathogens including bacteria and fungi are surrounded, in addition to the plasma membrane, by an external barrier, which contains mainly negative charge polysaccharide compounds. $^{37)}$ 
Furthermore, some dendrimers are emerging as the new topical antimicrobial agents - peptide dendrimers are effective against 'herpes simplex virus' and the polylysine (SPL7013) dendrimer is currently undergoing Phase II human clinical trials to test its efficacy. ${ }^{38)}$ Different approaches have been used to enhance the antimicrobial activities of dendrimers and the most conventional being carriers for the antimicrobial agents. PAMAM dendrimers as quinolone carriers were active and useful for topical delivery of microbicides. ${ }^{39)}$ The silver complexes and nanocomposites with PAMAM dendrimer resulted in increased antibacterial activity towards the Staphylococcus aureus, Pseudomonas aeruginosa and Escherichia coli due to the improved contact of microbes with the organized silver domains. ${ }^{40)}$

These encouraging results emphasize the potential usefulness of PAMAM dendrimers as carriers of antifungal agents and provide further impetus to synthesize and evaluate dendritic polymers for use in basic drug delivery studies and to design semisolid dosage forms based on dendrimers with antimicrobial drugs, like CLO. Evaluation of hydrogels containing CLO and PAMAM dendrimers is underway and in vitro microbiological activity and in vivo toxicity studies will be described in a due course.

\section{REFERENCES}

1) Sawyer P. R., Brogden R. N., Pinder R. M., Speight T. M., Avery G. S., Drugs, 9, 424-447 (1975).

2) Ellepola A. N., Samaranayake L. P., Martin M. V., "Antifungal Agents," Vol. I, ed. by Samaranayake L. P., MacFarlane T. W., WrightButterworth and Co., U.K., 1990.

3) Henry K. W., Nickels J. T., Edlind T. D., Antimicrob. Agents Chemother., 44, 2693-2700 (2000).

4) Vazquez J. A., HIV Clin. Trials, 1, 47-59 (2000).

5) Abdel-Moety E. M., Khattab F. I., Kelani K. M., Abou Al Alamein A. M., Farmaco, 57, 931-938 (2002).

6) Ning M., Gu Z., Pan H., Yu H., Xiao K., Indian J. Exp. Biol., 43, $150-157$ (2005).

7) Prabagar B., Yoo B. K., Woo J. S., Kim J. A., Rhee J. D., Piao M. G., Choi H. G., Yong C. S., Arch. Pharm. Res., 30, 249-254 (2007).

8) Chauhan A. S., Jain N. K., Diwan P. V., Khopade A. J., J. Drug Target., 12, 575-583 (2004).

9) Devarakonda B., Hill R. A., Liebenberg W., Brits M., de Villiers M. M., Int. J. Pharm., 304, 193-209 (2005).

10) Kolhe P., Misra E., Kannan R. M., Kannan S., Lieh-Lai M., Int. J. Pharm., 259, 143-160 (2003).

11) Tomalia D. A., Aldrichim. Acta, 2, 39-57 (2004).

12) Esfand R., Tomalia D. A., Drug Discov. Today, 6, 427-436 (2001).

13) Milhem O. M., Myles C., McKeown N. B., Attwood D., D'Emanuele A., Int. J. Pharm., 197, 239-241 (2000).

14) Gardiner J., Freeman S., Leach M., Green A., Alcock J., D'Emanuele A., J. Enzyme Inhib. Med. Chem., 23, 623-628 (2008).

15) Tulu M., Aghatabay N. M., Senel M., Dizman C., Parali T., Dulger B.,
Eur. J. Med. Chem., 44, 1093-1099 (2009).

16) Hájková R., Sklenářová H., Matysová L., Švecová P., Solich P., Talanta, 73, 483-489 (2007).

17) Hashiguchi T., Kodama A., Ryu A., Otagini M., Int. J. Pharm., 161, 195-204 (1998).

18) De Hoog G. S., Guarro J., "Atlas of Clinical Fungi," 1st ed., Centraalbureau voor Schimmelcultures, Baarn and Delft, the Netherlands, 1995.

19) Holt R. J., Postgrad. Med. J., 1, 24-26 (1974).

20) Clinical and Laboratory Standards Institute (CLSI), "Reference Method for Broth Dilution Antifungal Susceptibility Testing of Yeast, Approved Standard," 3rd ed., CLSI document M27-A3, Clinical and Laboratory Standards Institute, Wayne, PA, 2008.

21) Clinical and Laboratory Standards Institute (CLSI), "Reference Method for Broth Dilution Antifungal Susceptibility Testing of Yeast, Third Informational Supplement," CLSI document M27-S3, Clinical and Laboratory Standards Institute, Wayne, PA, 2008.

22) Cantón E., Pemán J., Viudes A., Quindós G., Gobernado M., EspinelIngroff A., Diagn. Microbiol. Infect. Dis., 45, 203-206 (2003).

23) Pedersen M., Bjerregaard S., Jacobson J., Sorensen A. M., Int. J. Pharm., 176, 121-131 (1998).

24) Najlah M., D’Emanuele A., Curr. Opin. Pharmacol., 6, 522-527 (2006).

25) Devarakonda B., Hill R. A., de Villiers M. M., Int. J. Pharm., 284, 133-140 (2004).

26) Ma M., Cheng Y., Xu Z., Xu P., Qu H., Fang Y., Xu T., Wen L., Eur. J. Med. Chem., 42, 93-98 (2007).

27) Gupta U., Agashe H. B., Asthana A., Jain N. K., Biomacromolecules, 7, 649-658 (2006).

28) Peschka M., Roberts P. H., Knepper T. P., Anal. Bioanal. Chem., 389, 959-968 (2007).

29) Winnicka K., Bielawski K., Rusak M., Bielawska A., J. Health Sci., 55, 169-177 (2009).

30) Lopez A. I., Reins R. Y., McDermott A. M., Trautner B. W., Cai C., Mol. Biosyst., 5, 1148-1156 (2009).

31) Perumal O. P., Inapagolla R., Kannan S., Kannan R. M., Biomaterials, 29, 3469-3476 (2008)

32) Hong S., Bielinska A. U., Mecke A., Keszler B., Beals J. L., Shi X., Balogh L., Orr B. G., Baker J. R. Jr., Banaszak Holl M. M., Bioconjug. Chem., 15, 774-782 (2004).

33) Klajnert B., Janiszewska J., Urbanczyk-Lipkowska Z., Bryszewska M., Epand R. M., Int. J. Pharm., 327, 145-152 (2006).

34) Gardikis K., Hatziantoniou S., Viras K., Wagner M., Demetzos C., Int. J. Pharm., 318, 118-123 (2006).

35) Leroueil P. R., Berry S. A., Duthie K., Han G., Rotello V. M., McNerny D. Q., Baker J. R. Jr., Orr B. G., Banaszak Holl M. M., Nano Lett., 8, 420-424 (2008).

36) Arnold L. J. Jr., Dagan A., Gutheil J., Kaplan N. O., Proc. Natl. Acad. Sci. U.S.A., 76, 3246-3250 (1979).

37) Klis F. M., de Groot P., Hellingwerf K., Med. Mycol., 39 (Suppl. 1), $1-8$ (2001).

38) Mumper R. J., Bell M. A., Worthen D. R., Cone R. A., Lewis G. R., Paull J. R., Moench T. R., Drug Dev. Ind. Pharm., 35, 515-524 (2009).

39) Cheng Y., Qu H., Ma M., Xu Z., Xu P., Fang Y., Xu T., Eur. J. Med. Chem., 42, 1032-1038 (2007).

40) Balogh L., Swanson D. R., Tomalia D. A., Hagnauer G. L., McManus A. T., Nano Lett., 1, 18-21 (2001). 